\title{
Surface Polymer Network Model and Effective Membrane Curvature Elasticity
}

\author{
R.Podgornik* \\ Laboratory of Structural Biology \\ Division of Computer Research and Technology \\ National Institutes of Health, Bethesda, MD 20892
}

\begin{abstract}
A microscopic model of a surface polymer network - membrane system is introduced, with contact polymer surface interactions that can be either repulsive or attractive and sliplinks of functionality four randomly distributed over the supporting membrane surface anchoring the polymers to it. For the supporting surface perturbed from a planar configuration and a small relative number of surface sliplinks, we investigate an expansion of the free energy in terms of the local curvatures of the surface and the surface density of sliplinks, obtained through the application of the Balian - Bloch - Duplantier multiple surface scattering method. As a result, the dependence of the curvature elastic modulus, the Gaussian modulus as well as of the spontaneous curvature of the "dressed" membrane, i.e. polymer network plus membrane matrix, is obtained on the mean polymer bulk end to end separation and the surface density of sliplinks.
\end{abstract}

PACS. 82.70 Disperse systems.

PACS. 05.20 Statistical Mechanics.

PACS. 61.25H Macromolecular and polymer solutions.

December 7, 2017

*On leave from J.Stefan Institute, P.O.B. 100, 61000 Ljubljana, SLOVENIA. 


\section{Introduction}

Polymer networks often provide highly specialized elastic properties to biological systems. Structure of the red blood cell (RBC) membrane is typical in this respect [1] in the sense that it unites the lipid matrix and the (polymer) spectrin network into a single "dressed" membrane, where the polymer network not only interacts with the underlying lipid surface but is actually anchored to it via ankyrin molecules. The polymer network consists of negatively charged spectrin tetramers of $\sim 200 \mathrm{~nm}$ contour length, with intrinsic persistence length of $\sim 10 \mathrm{~nm}$. The replicating network of spectrin tetramers has a junction functionality of between 4 and 6 . The network junctions do not coincide with attachment points (ankyrin molecules). The contour length of the spectrin tetramers is between $2-6$ times larger then the separation between anchoring points and the whole system thus looks like a crosslinked two dimensional gel where the crosslinks are bound to remain on the supporting lipid surface while the polymers are allowed to stretch into the cytoplasmic (polymer rich) solution and away from the lipid surface [2].

The problem of elastic properties of a "dressed" membrane, where the individual polymer molecules or their junctions are confined to lie on (or are embedded in) the supporting surface, motivated by our present understanding of the RBC spectrin network, received recently a lot of attention [3, 4, 5]. The method of choice in most of these investigations has been the Monte Carlo simulations that led to several important insights regarding surface - tethered polymers and/or surface polymer networks. The analytical limits for these systems have been much harder to come by due to the complicated nature of the polymer network - surface interactions. In this paper we shall try to fill this gap and establish some approximate limiting analytical results for the elastic properties of a surface polymer network pinned to a supporting flexible membrane surface. More specifically we shall investigate the modifications in the elastic energy parameters of a flexible membrane wrought by the presence of a surface polymer gel where the crosslinks (in this case presumed to be sliplinks) are constrained to lie on the supporting membrane surface. The parts of the polymeric chains between the junctions are allowed to sample all the configurations on one side of the supporting membrane, i.e. are constrained to lie in the halfspace defined by the position of the membrane surface (see Fig.1).

Several theoretical studies lately addressed the issues pertaining to the modifications of elastic properties of a membrane in contact with a polymer solution that interacts with it [6, 7, 8]. Though the problem in this case is simpler than in the surface polymer gel case, it appears that certain features of the approach employed [8] can be generalized to this case. Specif- 
ically we shall derive the curvature expansion of the free energy of a surface polymer gel with deformed supporting surface and show that the spontaneous curvature as well as the elastic modulus of the "dressed" membrane become functions of the polymer parameters, most notably the monomer surface interaction and the polymer bulk end - end separation.

We presume that the chains in between the junctions are Gaussian and that their interaction with the supporting membrane surface, aside from the sliplinks where they are pinned to the surface, is of a contact type, thus simulating the screened electrostatic or other short range interactions between polymer segments and the membrane surface (see Fig.1). The proposed formulation of the surface gel on a non - planar supporting surface problem is closely related to the formulation of similar polymer problems in the bulk introduced by Edwards and Freed [9] and is particularly suited for the application of the Balian - Bloch - Duplantier [10] multiple surface scattering Green function formalism, which gives a local curvature expansion for a Helmholtz equation in a region with deformed boundaries.

A major drawback of this approach is that we have been unable to include volume interactions between polymer chains, extensively studied in [3, :4], into the formulation in a consistent manner. However, this drawback is substantially counterbalanced by the fact that first of all analytic results are feasible in this approximation and that the effect of even very complicated polymer - surface interactions, as is the case in this model system, can be readily investigated within the developed formalism.

\section{Model}

We define our system as a statistical ensemble of freely mobile sliplinks confined to the supporting surface $\mathbf{r}_{\alpha}$ (here and below the Greek indices stand for coordinates along the supporting surface) produced after $N$ chains $\mathcal{N}$ monomers long are crosslinked $M$ times with functionality four. Following [9] the crosslinks are taken to be introduced by an external auxiliary field, $\phi(\mathbf{r})$, that produces polymer chains with Green function $\mathcal{G}_{\phi}\left(\mathbf{R}, \mathbf{R}^{\prime} ; \mathcal{N}\right)$ if the chains are $\mathcal{N}$ segments long. One can show through a diagrammatic expansion of this Green function that if it is averaged over a Gaussian distribution of $\phi(\mathbf{r})$ this operation formally introduces an arbitrary number of cross-links of functionality four, distributed randomly through space and along the chains, 
with the partition function

$$
\Xi(N, \mathcal{N})=\left\langle\left(\iint d^{3} \mathbf{R} \mathcal{G}_{\phi}\left(\mathbf{R}, \mathbf{R}^{\prime} ; \mathcal{N}\right) d^{3} \mathbf{R}^{\prime}\right)^{N}\right\rangle_{\phi}
$$

for $N$ chains.

If one now expands the Green function in terms of $\sqrt{\mu} \phi(\mathbf{r})$ and then averages with $\langle\ldots\rangle_{\phi}$, the coefficient of $\mu^{M}$ contains all the terms with $M$ crosslinks randomly distributed across the space and along the chains. Thus the partition function of a system with $M$ crosslinks can be formally written as

$$
\Xi(M, N, \mathcal{N})=\frac{1}{2 \pi \imath} \oint_{\mathcal{C}} \frac{M ! d \mu}{\mu^{M+1}}\left\langle\left(\iint d^{3} \mathbf{R} \mathcal{G}_{\phi}\left(\mathbf{R}, \mathbf{R}^{\prime} ; \mathcal{N}\right) d^{3} \mathbf{R}^{\prime}\right)^{N}\right\rangle_{\phi}
$$

For the system discussed here the partition function can be obtained along the lines of [9], except that the auxiliary field should now be confined to the surface $\mathbf{r}_{\alpha}$. This would signify that it should effect not the equation defining the Green function itself, but rather its boundary condition at this surface. Also if there exist other interactions between the polymers and the surface, they should also show up only in the boundary condition if their range is short enough.

Putting now everything together we derive the partition function of our model system in the following compact notation

$\Xi(M, N, \mathcal{N})=\frac{M !}{2 \pi \imath} \oint_{\mathcal{C}} \frac{d \mu}{\mu^{M+1}}\left\langle\left[\mathcal{L}^{-1} \iint d^{3} \mathbf{r} d^{3} \mathbf{r}^{\prime} \mathcal{G}_{\phi}\left(\mathbf{r}, \mathbf{r}^{\prime} ; s\right)\right]^{N}\right\rangle_{\phi}=\frac{M !}{2 \pi \imath} \oint_{\mathcal{C}} \frac{d \mu}{\mu^{M+1}}\left\langle\Xi_{\phi}(\mu, \mathcal{N})^{N}\right\rangle_{\phi}$,

where $\mathcal{G}_{\phi}\left(\mathbf{r}, \mathbf{r}^{\prime} ; s\right)$ is the Laplace transformed Green function of a free flight polymer, satisfying a Helmholtz - type equation [1]

$$
\left[\nabla^{2}-s\right] \mathcal{G}_{\phi}\left(\mathbf{r}, \mathbf{r}^{\prime} ; s\right)=-\delta^{3}\left(\mathbf{r}-\mathbf{r}^{\prime}\right)
$$

Here we made a transformation $\mathbf{r} \rightarrow \frac{\sqrt{6} \mathbf{r}}{\ell}$, measuring the spatial dimensions in units of $\frac{\ell}{\sqrt{6}}$, where $\ell$ is the steplength. $\mathcal{L}^{-1}$ stands for the inverse Laplace transform, i.e. $\mathcal{L}^{-1} f(s)=\oint_{\mathcal{C}} e^{s \mathcal{N}} f(s) d s=f(\mathcal{N})$ and the definition of $\Xi_{\phi}(\mu, \mathcal{N})$ is obvious. At the supporting membrane surface $\mathbf{r}_{\alpha}$ the Green function satisfies [12]

$$
\frac{\partial \mathcal{G}_{\phi}\left(\mathbf{r}_{\alpha}, \mathbf{r}^{\prime} ; s\right)}{\partial \mathbf{n}_{\alpha}}-\kappa \mathcal{G}_{\phi}\left(\mathbf{r}_{\alpha}, \mathbf{r}^{\prime} ; s\right)+\sqrt{\mu} \phi\left(\mathbf{r}_{\alpha}\right) \mathcal{G}_{\phi}\left(\mathbf{r}_{\alpha}, \mathbf{r}^{\prime} ; s\right)=0
$$

where $\kappa$ is the strength of the contact membrane surface - polymer interaction. It can be either repulsive $(\kappa>0)$ or attractive $(\kappa<0)$. The last 
term in the above equation, proportional to $\sqrt{\mu}$, creates at random positions along the supporting surface $\mathbf{r}_{\alpha}$ as well as along the polymer chains junctions of fourfold functionality (sliplinks), of chemical potential $\mu$ per single junction, after the Green function is averaged over a Gaussian distribution of the auxiliary field $\phi\left(\mathbf{r}_{\alpha}\right)$ [9],

$$
\langle\ldots\rangle_{\phi}=\int \mathcal{D} \phi\left(\mathbf{r}_{\alpha}\right)(\ldots) e^{-\frac{1}{2} \int \phi^{2}\left(\mathbf{r}_{\alpha}\right) d^{2} \mathbf{r}_{\alpha}} / \int \mathcal{D} \phi\left(\mathbf{r}_{\alpha}\right) e^{-\frac{1}{2} \int \phi^{2}\left(\mathbf{r}_{\alpha}\right) d^{2} \mathbf{r}_{\alpha}} .
$$

The averaging over a Gaussian distribution of the auxiliary field preserves only terms with even powers of $\sqrt{\mu}$.

We shall now try to obtain an approximate solution of the above model in the limit of $\frac{M}{N} \ll 1$ while separately $N \gg 1$ and $M \gg 1$, with the additional proviso that the volume (or surface) density of chains $\frac{N}{V}$ (or $\frac{N}{S_{\omega}}$ depending on the sign of $\kappa$ ) and the surface density of sliplinks $\frac{M}{S_{\omega}}$ are finite (thermodynamic limit).

We first of all cast the model in a form that will be appropriate for later formal developements. It is easiest to obtain a power series solution of Eq.5 in terms of the free space solution $\mathcal{G}_{0}\left(\mathbf{r}, \mathbf{r}^{\prime} ; s\right)$ i.e.

$$
\mathcal{G}_{0}\left(\mathbf{r}, \mathbf{r}^{\prime} ; s\right)=\frac{e^{-\sqrt{s}\left|\mathbf{r}-\mathbf{r}^{\prime}\right|}}{4 \pi\left|\mathbf{r}-\mathbf{r}^{\prime}\right|}
$$

This series has been already derived by Balian and Bloch in [10] and we merely quote their result. Introducing $\Gamma\left(\mathbf{r}_{\gamma}, \mathbf{r}_{\epsilon} ; s\right)$ as

$$
\Gamma\left(\mathbf{r}_{\alpha}, \mathbf{r}_{\beta} ; s\right)+2 \kappa \int d S_{\gamma} \mathcal{G}_{0}\left(\mathbf{r}_{\alpha}, \mathbf{r}_{\gamma} ; s\right) \Gamma\left(\mathbf{r}_{\gamma}, \mathbf{r}_{\beta} ; s\right)=\delta^{2}\left(\mathbf{r}_{\alpha}-\mathbf{r}_{\beta}\right),
$$

one obtains the following expansion for the Green function

$$
\begin{aligned}
\mathcal{G}_{\phi}\left(\mathbf{r}, \mathbf{r}^{\prime} ; s\right) & =\mathcal{G}_{0}(\mathbf{r}, \mathbf{r} ; s)+2 \iint d S_{\alpha} d S_{\beta} \mathcal{G}_{0}\left(\mathbf{r}, \mathbf{r}_{\alpha} ; s\right) \Gamma\left(\mathbf{r}_{\alpha}, \mathbf{r}_{\beta} ; s\right) \nabla_{\beta} \mathcal{G}_{0}\left(\mathbf{r}_{\beta}, \mathbf{r}^{\prime} ; s\right)+ \\
& +2^{2} \iiint \int d S_{\alpha} d S_{\beta} d S_{\gamma} d S_{\delta} \mathcal{G}_{0}\left(\mathbf{r}, \mathbf{r}_{\alpha} ; s\right) \Gamma\left(\mathbf{r}_{\alpha}, \mathbf{r}_{\delta} ; s\right) \frac{\partial \mathcal{G}_{0}\left(\mathbf{r}_{\delta}, \mathbf{r}_{\gamma} ; s\right)}{\partial \mathbf{n}_{\delta}} \Gamma\left(\mathbf{r}_{\delta}, \mathbf{r}_{\beta} ; s\right) \nabla_{\beta} \mathcal{G}_{0}\left(\mathbf{r}_{\beta}, \mathbf{r}^{\prime} ; s\right)+\ldots
\end{aligned}
$$

where

$$
\nabla_{\alpha}=\frac{\partial}{\partial \mathbf{n}_{\alpha}}-\kappa+\sqrt{\mu} \phi\left(\mathbf{r}_{\alpha}\right) .
$$

We might just add here that for a planar surface the normal derivative of the Green function, $\frac{\partial \mathcal{G}_{0}\left(\mathbf{r}_{\delta}, \mathbf{r}_{\gamma} ; s\right)}{\partial \mathbf{n}_{\delta}}$, is zero and thus the expansion Eq.9 is basically an expansion in the curvature of the bounding surface. 
Combining now both Eqs.8 and 9 we can derive the partition function in a rather transparent form

$$
\begin{aligned}
\Xi_{\phi}(\mu, \mathcal{N}) & =\Xi_{b}(\mathcal{N})+2 \operatorname{Tr} U_{\phi}\left(\mathbf{r}_{\alpha}, \mathbf{r}_{\beta} ; \mathcal{N}\right)= \\
& =V+2 \int d S_{\omega} U_{\phi}\left(\mathbf{r}_{\omega}, \mathbf{r}_{\omega} ; \mathcal{N}\right),
\end{aligned}
$$

where $\Xi_{b}(\mathcal{N})=\iint d^{3} \mathbf{r} d^{3} \mathbf{r}^{\prime} \mathcal{G}_{0}\left(\mathbf{r}, \mathbf{r}^{\prime} ; \mathcal{N}\right)=V$ is the volume of the polymer solution with unperturbed boundaries and the operator $U_{\phi}\left(\mathbf{r}_{\alpha}, \mathbf{r}_{\beta} ; s\right)$ is obtained as a solution of

$$
U_{\phi}\left(\mathbf{r}_{\alpha}, \mathbf{r}_{\beta} ; s\right)-2 \int d S_{\gamma} U_{\phi}\left(\mathbf{r}_{\alpha}, \mathbf{r}_{\gamma} ; s\right) \nabla_{\gamma} \mathcal{G}_{0}\left(\mathbf{r}_{\gamma}, \mathbf{r}_{\beta} ; s\right)=\nabla_{\alpha} F\left(\mathbf{r}_{\alpha}, \mathbf{r}_{\beta} ; s\right),
$$

with $d S_{\omega}$ being the area element of the surface $\mathbf{r}_{\alpha}$, and

$$
F\left(\mathbf{r}_{\alpha}, \mathbf{r}_{\beta} ; s\right)=\iint d^{3} \mathbf{r} d^{3} \mathbf{r}^{\prime} \mathcal{G}_{0}\left(\mathbf{r}, \mathbf{r}_{\alpha} ; s\right) \mathcal{G}_{0}\left(\mathbf{r}_{\beta}, \mathbf{r}^{\prime} ; s\right)
$$

Eqs.11 and 12 represent just a more compact formalisation of the results already derived in 10 that is conveniently suited for our purposes.

Formally the above equations define our model system. Their solution is obviously non-trivial and additional approximations have to be introduced in order to make the problem tractable. First of all we shall introduce the approxiation of low polymer density that will make the evaluation of $\phi(\mathbf{r})$ average tractable in the form of a power series in the chemical potential of the sliplinks, $\mu$. Next we shall resolve the integration over $\mu$ by reverting to the saddle - point ansatz and finally we shall use the approximation of the local tangential plane that explicitely introduces the surface curvature dependence of the different Green functions to evaluate the different curvature terms in the final expression for the partition function.

\section{Analysis}

Starting from Eq.11, $\Xi_{\phi}(\mu, \mathcal{N})^{N}$ can be written in the form of a "virial" expansion in terms of the polymer volume density. If this density is small enough we can stop at the first order term thus obtaining

$\Xi(M, N, \mathcal{N}) \cong \frac{M !}{2 \pi \imath} \oint_{\mathcal{C}} \frac{d \mu}{\mu^{M+1}} e^{N \ln \left\langle\Xi_{\phi}(\mu, \mathcal{N})\right\rangle_{\phi}}=\frac{M !}{2 \pi \imath} \oint_{\mathcal{C}} \frac{d \mu}{\mu^{M+1}} e^{N \ln \left(V+2 \operatorname{Tr}\left\langle U_{\phi}\left(\mathbf{r}_{\alpha}, \mathbf{r}_{\beta} ; \mathcal{N}\right)\right\rangle_{\phi}\right)}$.

As we have done before [8] we now substantially reduce the heavy algebra proceeding from the above equations if we presume that $|\kappa| \gg$ 
$\frac{\partial \log \mathcal{G}_{0}\left(\mathbf{r}_{\alpha}, \mathbf{r}^{\prime} ; s\right)}{\partial \mathbf{n}_{\alpha}}$. First of all we solve Eq.12 in the form of a formal series in $\phi\left(\mathbf{r}_{\alpha}\right)$. This series solution of Eq.12 for $U_{\phi}\left(\mathbf{r}_{\alpha}, \mathbf{r}_{\beta} ; s\right)$ can be effectively resummed and averaged over the Gaussian distribution of the auxiliary field $\phi\left(\mathbf{r}_{\alpha}\right)$ Eq.6. Only even powers of $\phi\left(\mathbf{r}_{\alpha}\right)$ survive this averaging and we can regroup the remaining terms obtaining a power series in $\mu$. By defining

$$
\begin{aligned}
U_{0}\left(\mathbf{r}_{\alpha}, \mathbf{r}_{\beta} ; s\right) & =-\kappa \int d S_{\gamma} F\left(\mathbf{r}_{\alpha}, \mathbf{r}_{\gamma} ; s\right) \Gamma\left(\mathbf{r}_{\gamma}, \mathbf{r}_{\beta} ; s\right) \\
\Delta\left(\mathbf{r}_{\alpha}, \mathbf{r}_{\beta} ; s\right) & =\int d S_{\gamma} \mathcal{G}_{0}\left(\mathbf{r}_{\alpha}, \mathbf{r}_{\gamma} ; s\right) \Gamma\left(\mathbf{r}_{\gamma}, \mathbf{r}_{\beta} ; s\right) \\
W_{0}\left(\mathbf{r}_{\alpha}, \mathbf{r}_{\beta} ; s\right) & =\frac{1}{2} \iint d S_{\gamma} d S_{\epsilon} F\left(\mathbf{r}_{\alpha}, \mathbf{r}_{\gamma} ; s\right) \Gamma\left(\mathbf{r}_{\gamma}, \mathbf{r}_{\epsilon} ; s\right) \mathcal{G}_{0}^{-1}\left(\mathbf{r}_{\epsilon}, \mathbf{r}_{\beta} ; s\right)
\end{aligned}
$$

we thus obtain the $\phi$ average of $U_{\phi}\left(\mathbf{r}_{\alpha}, \mathbf{r}_{\beta} ; s\right)$ in the form of a series in the chemical potential of the pinning sliplinks $\mu$

$$
\begin{aligned}
\left\langle U_{\phi}\left(\mathbf{r}_{\alpha}, \mathbf{r}_{\beta} ; s\right)\right\rangle_{\phi} & =U_{0}\left(\mathbf{r}_{\alpha}, \mathbf{r}_{\beta} ; s\right) \\
& +4 \mu \int d S_{\gamma} \Delta\left(\mathbf{r}_{\alpha}, \mathbf{r}_{\gamma} ; s\right) W_{0}\left(\mathbf{r}_{\gamma}, \mathbf{r}_{\beta} ; s\right)+ \\
& +16 \mu^{2} \iint d S_{\gamma} d S_{\epsilon} \Delta\left(\mathbf{r}_{\alpha}, \mathbf{r}_{\epsilon} ; s\right) \Delta\left(\mathbf{r}_{\epsilon}, \mathbf{r}_{\gamma} ; s\right) W_{0}\left(\mathbf{r}_{\gamma}, \mathbf{r}_{\beta} ; s\right)+\ldots
\end{aligned}
$$

where we redefined the chemical potential $\mu \rightarrow \mu \Delta\left(\mathbf{r}_{\alpha}, \mathbf{r}_{\alpha} ; \mathcal{N}\right)$, with $\Delta\left(\mathbf{r}_{\alpha^{-}}\right.$ , $\left.\mathbf{r}_{\alpha} ; \mathcal{N}\right) \rightarrow \pi \sqrt{\mathcal{N}} V^{-\frac{1}{3}} \gg 1$. This relation stemms from the conservation of the degrees of freedom of the chain [9]. At each order of the $\mu$ expansion we retain only the largest term while omitting all the additional powers of $\Delta^{-1}\left(\mathbf{r}_{\alpha}, \mathbf{r}_{\alpha} ; \mathcal{N}\right)$. The above expansion in terms of $\mu$ is convergent if $\frac{M}{N} \ll 1$, and we can stop at the second order term.

The zero order term in this expansion, Eq.16, is exactly the one corresponding to the example treated previously [8], which corresponds to the case without any surface pinning of the polymers through the sliplinks.

The approximate form of $\left\langle\Xi_{\phi}(\mu, \mathcal{N})\right\rangle_{\phi}$ is thus obtained as

$$
\left\langle\Xi_{\phi}(\mu, \mathcal{N})\right\rangle_{\phi}=V+2 \int d S_{\omega}\left\langle U_{\phi}\left(\mathbf{r}_{\omega}, \mathbf{r}_{\omega} ; \mathcal{N}\right)\right\rangle_{\phi} \cong \Xi_{0}(\mathcal{N})+\mu \Xi_{1}(\mathcal{N})+\frac{1}{2} \mu^{2} \Xi_{2}(\mathcal{N})+\ldots
$$

where aside from $V$ all the other $\mu$ terms in the expansion can be obtained by comparison with Eq.16 as

$$
\begin{aligned}
& \Xi_{0}(\mathcal{N})=\Xi_{b}(\mathcal{N})+2 \mathcal{L}^{-1}\left[\operatorname{Tr} U_{0}\left(\mathbf{r}_{\alpha}, \mathbf{r}_{\beta} ; s\right)\right] \\
& \Xi_{1}(\mathcal{N})=8 \mathcal{L}^{-1}\left[\operatorname{Tr} \int d S_{\gamma} \Delta\left(\mathbf{r}_{\alpha}, \mathbf{r}_{\gamma} ; s\right) W_{0}\left(\mathbf{r}_{\gamma}, \mathbf{r}_{\beta} ; s\right)\right] \\
& \Xi_{2}(\mathcal{N})=64 \mathcal{L}^{-1}\left[\operatorname{Tr} \iint d S_{\gamma} d S_{\epsilon} \Delta\left(\mathbf{r}_{\alpha}, \mathbf{r}_{\gamma} ; s\right) \Delta\left(\mathbf{r}_{\gamma}, \mathbf{r}_{\epsilon} ; s\right) W_{0}\left(\mathbf{r}_{\epsilon}, \mathbf{r}_{\beta} ; s\right)\right]
\end{aligned}
$$


where $\Xi_{b}(\mathcal{N})=\mathcal{L}^{-1}\left[\frac{1}{s} \int d^{3} \mathbf{r}\right]=V$.

The final $\mu$ integration in Eq. 14 is now of the form

$$
\Xi(M, N, \mathcal{N}) \cong \frac{M !}{2 \pi \imath} \oint_{\mathcal{C}} d \mu e^{N \ln \left(\Xi_{0}(\mathcal{N})+\mu \Xi_{1}(\mathcal{N})+\frac{1}{2} \mu^{2} \Xi_{2}(\mathcal{N})+\ldots\right)-(M+1) \ln \mu}
$$

which is amenable to an analytic treatment through the saddle - point approximation in the thermodynamic limit of $N \gg 1$ and $M \gg 1$, yielding an approximate result $\mu=\mu^{*}\left(\frac{M}{N}\right)$, where to the lowest order we obtain $\mu^{*} \sim \frac{M}{N} \frac{\Xi_{0}(\mathcal{N})}{\Xi_{1}(\mathcal{N})}+\ldots$ The saddle - point free energy $\mathcal{F}$ is thus obtained as $\left(\beta=(k T)^{-1}\right)$

$$
\begin{aligned}
\beta \mathcal{F} & =-\ln \Xi(M, N, \mathcal{N}) \cong \\
& \cong-N \ln \left(\Xi_{0}(\mathcal{N})+\mu^{*} \Xi_{1}(\mathcal{N})+\frac{1}{2} \mu^{* 2} \Xi_{2}(\mathcal{N})\right)+(M+1) \ln \mu^{*}
\end{aligned}
$$

Following the expansion of the statistical sum in terms of the relative number of sliplinks, i.e. $\frac{M}{N}$, we now additionally presume that the supporting surface $\mathbf{r}_{\alpha}$ is deformed and we investigate the lowest order expansion of the free energy in terms of the local curvatures of the deformed surface. We approach this problem by the Balian - Bloch - Duplantier method [10] as was explained in detail for a somewhat simpler case of polymer - surface interactions [8].

The principle of the curvature expansion is simple. First of all we note that the partition function Eq.18 contains different surface integrals of either $G_{0}\left(\mathbf{r}_{\alpha}, \mathbf{r}_{\beta} ; s\right), \Delta\left(\mathbf{r}_{\alpha}, \mathbf{r}_{\beta} ; s\right)$ or $F\left(\mathbf{r}_{\alpha}, \mathbf{r}_{\beta} ; s\right)$. Since all these quantities depend only on the difference of the surface coordinates in the arguments we can expand them around the local tangential plane up to the second order in the deviations from that plane and then evaluate the surface integrals explicitely. This expansion in terms of the deviations from the local tangential plane is valid only as long as the Green function is of a range smaller than the local curvatures. Practically this would mean that one can always find a range of curvature values where the expansion of a Yukawa - type Green function Eq.] in the vicinity of a local tangential plane of a non-planar surface is justified.

We write the equation of the bounding surface $\mathbf{r}_{\alpha}$ in the reference frame of the tangential plane at $\mathbf{r}_{\omega}$ as

$$
z_{\omega}(x, y) \cong \frac{1}{2}\left(\frac{x^{2}}{R_{\omega_{1}}}+\frac{y^{2}}{R_{\omega_{2}}}\right)+\ldots
$$

where $R_{\omega_{1}}$ and $R_{\omega_{2}}$ are the two principal radii of curvature at the point $\mathbf{r}_{\omega}$ on the bounding surface. 
We can now straightforwardly derive the form of the curvature dependence up to and including second order of the Green function by expanding the coordinate dependence in the vicinity of tangential plane for each point along the bounding surface. The only non-zero terms in the expansion of $G_{0}\left(\mathbf{r}_{\alpha}, \mathbf{r}_{\beta} ; s\right)$ are the zero and the second order, thus giving

$$
G_{0}\left(\mathbf{r}_{\alpha}, \mathbf{r}_{\beta} ; s\right) \cong \stackrel{(p)}{G_{0}}\left(\mathbf{r}_{\alpha}, \mathbf{r}_{\beta} ; s\right)+\frac{1}{2} z_{\alpha}^{2}(x, y) \frac{\partial^{2} \stackrel{(p)}{G_{0}}\left(\mathbf{r}_{\alpha}, \mathbf{r}_{\beta} ; s\right)}{\partial z_{\alpha}^{2}}+\ldots
$$

where the superscript $p$ stands for 'planar' (i.e. zero curvature) approximation of the superscripted quantity. Furthermore $\frac{\partial G_{0}\left(\mathbf{r}_{\alpha}, \mathbf{r}_{\beta} ; s\right)}{\partial \mathbf{n}_{\alpha}}$ obviously has only odd terms in the expansion starting with

$$
\frac{\partial G_{0}\left(\mathbf{r}_{\alpha}, \mathbf{r}_{\beta} ; s\right)}{\partial \mathbf{n}_{\alpha}} \cong-\left(\frac{\partial z_{\alpha}(x, y)}{\partial x} \frac{\partial}{\partial x}+\frac{\partial z_{\alpha}(x, y)}{\partial y} \frac{\partial}{\partial y}\right) \stackrel{(p)}{G_{0}}\left(\mathbf{r}_{\alpha}, \mathbf{r}_{\beta} ; s\right)+\ldots
$$

since the local normal is defined as $\mathbf{n}_{\alpha}=\left(1,-\frac{\partial z_{\alpha}(x, y)}{\partial x},-\frac{\partial z_{\alpha}(x, y)}{\partial y}\right)$. The third origin of the curvature dependence is the surface area element $d S_{\omega}$ itself in Eqs.8 and 15

$$
d S_{\omega} \cong d \stackrel{(p)}{S}_{\omega}+\frac{1}{2}\left(\nabla z_{\omega}(x, y)\right)^{2} d \stackrel{(p)}{S}{ }_{\omega} .
$$

Finally expressions Eqs.22, 23 and 24 have to be averaged locally over all the directions of the principal curvature axes.

These fundamental dependencies of the Green function $G_{0}\left(\mathbf{r}_{\alpha}, \mathbf{r}_{\beta} ; s\right)$ and its normal derivative on the local curvature now generate the corresponding functional dependence for the surface Green function $\Gamma\left(\mathbf{r}_{\alpha}, \mathbf{r}_{\beta} ; s\right)$ through the defining equation Eq.8 and for $F\left(\mathbf{r}_{\alpha}, \mathbf{r}_{\beta} ; s\right)$ that can be written in an alternative form

$$
\begin{aligned}
F\left(\mathbf{r}_{\alpha}, \mathbf{r}_{\beta} ; s\right) & =\iint d^{3} \mathbf{r} d^{3} \mathbf{r}^{\prime} G_{0}\left(\mathbf{r}, \mathbf{r}_{\alpha} ; s\right) G_{0}\left(\mathbf{r}_{\beta}, \mathbf{r}^{\prime} ; s\right)= \\
& =\frac{1}{s^{2}}\left[\frac{1}{4}-\int \frac{\partial G_{0}\left(\mathbf{r}_{\gamma}, \mathbf{r}_{\alpha} ; s\right)}{\partial \mathbf{n}_{\gamma}} d S_{\gamma}+\iint \frac{\partial G_{0}\left(\mathbf{r}_{\gamma}, \mathbf{r}_{\alpha} ; s\right)}{\partial \mathbf{n}_{\gamma}} \frac{\partial G_{0}\left(\mathbf{r}_{\gamma^{\prime}}, \mathbf{r}_{\beta} ; s\right)}{\partial \mathbf{n}_{\gamma^{\prime}}} d S_{\gamma} d S_{\gamma^{\prime}}\right]
\end{aligned}
$$

where the curvature dependence transpires more directly. With all these provisos we can now evaluate the surface integrals in the defining equations Eq.15 explicitely order by order in the inverse curvature [8]. Though this procedure is tedious it is nevertheless straightforward. The following relations are obtained

$$
\begin{aligned}
\int \stackrel{(0)}{U}\left(\mathbf{r}_{\omega}, \mathbf{r}_{\alpha} ; s\right) d \stackrel{(p)}{S} \alpha & =-\frac{\kappa}{4 \sqrt{s}^{3}(\kappa+\sqrt{s})} \\
\int \stackrel{(1)}{U}\left(\mathbf{r}_{\omega}, \mathbf{r}_{\alpha} ; s\right) d \stackrel{(p)}{S} \alpha & =\frac{\kappa}{2 s^{2}(\kappa+\sqrt{s})} \times \frac{1}{R_{\omega}}
\end{aligned}
$$




$$
\begin{aligned}
& \int \stackrel{(2)}{U}\left(\mathbf{r}_{\omega}, \mathbf{r}_{\alpha} ; s\right) d \stackrel{(p)}{S}{ }_{\alpha}=-\frac{\kappa}{4 \sqrt{s}^{5}(\kappa+\sqrt{s})} \times\left[\frac{1}{R_{\omega}^{2}}-\frac{\kappa}{2(\kappa+\sqrt{s})}\left(\frac{1}{R_{\omega}^{2}}-\frac{1}{R_{\omega_{1}} R_{\omega_{2}}}\right)\right] \\
& \int \stackrel{(0)}{\Delta}\left(\mathbf{r}_{\omega}, \mathbf{r}_{\alpha} ; s\right) d \stackrel{(p)}{S} \alpha=\frac{1}{2(\kappa+\sqrt{s})} \\
& \int \stackrel{(2)}{\Delta}\left(\mathbf{r}_{\omega}, \mathbf{r}_{\alpha} ; s\right) d \stackrel{(p)}{S} \alpha=\frac{1}{4 \sqrt{s}(\kappa+\sqrt{s})} \times\left(\frac{1}{R_{\omega}^{2}}-\frac{1}{R_{\omega_{1}} R_{\omega_{2}}}\right) \\
& \int \stackrel{(0)}{W}_{0}\left(\mathbf{r}_{\omega}, \mathbf{r}_{\alpha} ; s\right) d \stackrel{(p)}{S} \alpha=\frac{1}{4 s(\kappa+\sqrt{s})} \\
& \int \stackrel{(1)}{W}_{0}\left(\mathbf{r}_{\omega}, \mathbf{r}_{\alpha} ; s\right) d \stackrel{(p)}{S}=-\frac{1}{2 \sqrt{s}^{3}(\kappa+\sqrt{s})} \times \frac{1}{R_{\omega}} \\
& \int \stackrel{(2)}{W}_{0}\left(\mathbf{r}_{\omega}, \mathbf{r}_{\alpha} ; s\right) d \stackrel{(p)}{S} \alpha=\frac{1}{4 s^{2}(\kappa+\sqrt{s})} \times\left[\frac{1}{R_{\omega}^{2}}-\frac{(2 \kappa+\sqrt{s})}{2(\kappa+\sqrt{s})}\left(\frac{1}{R_{\omega}^{2}}-\frac{1}{R_{\omega_{1}} R_{\omega_{2}}}\right)\right]
\end{aligned}
$$

that are on the other hand the only quantities that we need in evaluation of the partition functions Eq.18. This being so due to the fact that $F\left(\mathbf{r}_{\alpha}, \mathbf{r}_{\beta} ; s\right)$ depends only on the difference between the coordinates and not on their absolute values. This property then reverberates through the defining equations all the way to the partition function Eq.18.

We now follow the definitions Eqs.16, 15 and 8 and thus as a result obtain a curvature expansion for $\Xi_{0}(\mathcal{N}), \Xi_{1}(\mathcal{N})$ and $\Xi_{2}(\mathcal{N})$. To the lowest order in the average curvature $\frac{1}{R_{\omega}}=\frac{1}{2}\left(\frac{1}{R_{\omega 1}}+\frac{1}{R_{\omega 2}}\right)$, and in the limit of long polymers, $\mathcal{N} \gg 1$, we derive the following curvature expansion for the case $\kappa>0$

$$
\begin{aligned}
& \Xi_{0}(\mathcal{N})=V-\frac{\sqrt{\mathcal{N}}}{\sqrt{\pi}} \int d S_{\omega}\left[1-\sqrt{\pi} \sqrt{\mathcal{N}} \frac{1}{R_{\omega}}+\frac{2 \mathcal{N}}{3}\left(\frac{1}{R_{\omega}^{2}}+\frac{1}{R_{\omega 1} R_{\omega 2}}\right)\right]+\ldots \\
& \Xi_{1}(\mathcal{N})=\frac{1}{\kappa^{2}} \int d S_{\omega}\left[1-\frac{4 \sqrt{\mathcal{N}}}{\sqrt{\pi}} \frac{1}{R_{\omega}}+\frac{\sqrt{\mathcal{N}}}{\kappa \sqrt{\pi}}\left(\frac{1}{R_{\omega}^{2}}-\frac{1}{R_{\omega 1} R_{\omega 2}}\right)+\mathcal{N} \frac{1}{R_{\omega 1} R_{\omega 2}}\right]+\ldots \\
& \Xi_{2}(\mathcal{N})=\frac{4}{\kappa^{3}} \int d S_{\omega}\left[1-\frac{4 \sqrt{\mathcal{N}}}{\sqrt{\pi}} \frac{1}{R_{\omega}}+\frac{2 \sqrt{\mathcal{N}}}{\kappa \sqrt{\pi}}\left(\frac{1}{R_{\omega}^{2}}-\frac{1}{R_{\omega 1} R_{\omega 2}}\right)+\mathcal{N} \frac{1}{R_{\omega 1} R_{\omega 2}}\right]+\ldots
\end{aligned}
$$

where $\omega$ stands for the index of the coordinate over which the final integration of the $\operatorname{Tr}$ operation is carried out, see Eq.17.

In the opposite case of $\kappa<0$ the spectrum of $G_{0}\left(\mathbf{r}_{\alpha}, \mathbf{r}_{\beta} ; \mathcal{N}\right)$ has bound states leading to the following dependence on $\mathcal{N} \gg 1$ after the inverse Laplace transform

$\Xi_{0}(\mathcal{N})=V+\frac{e^{\kappa^{2} \mathcal{N}}}{\kappa} \int d S_{\omega}\left[1-\frac{2}{\kappa} \frac{1}{R_{\omega}}+\mathcal{N}\left(\frac{1}{R_{\omega}^{2}}-\frac{1}{R_{\omega_{1}} R_{\omega_{2}}}\right)\right]+\ldots$ 


$$
\begin{aligned}
& \Xi_{1}(\mathcal{N})=\frac{4\left(\kappa^{2} \mathcal{N}\right) e^{\kappa^{2} \mathcal{N}}}{\kappa^{2}} \int d S_{\omega}\left[1-\frac{2}{\kappa} \frac{1}{R_{\omega}}+\frac{1}{\kappa^{2}} \frac{1}{R_{\omega}^{2}}+\mathcal{N}\left(\frac{1}{R_{\omega}^{2}}-\frac{1}{R_{\omega_{1}} R_{\omega_{2}}}\right)\right]+\ldots \\
& \Xi_{1}(\mathcal{N})=\frac{16\left(\kappa^{2} \mathcal{N}\right)^{2} e^{\kappa^{2} \mathcal{N}}}{\kappa^{3}} \int d S_{\omega}\left[1-\frac{2}{\kappa} \frac{1}{R_{\omega}}+\frac{1}{\kappa^{2}} \frac{1}{R_{\omega}^{2}}+\mathcal{N}\left(\frac{1}{R_{\omega}^{2}}-\frac{1}{R_{\omega_{1}} R_{\omega_{2}}}\right)\right]+\ldots
\end{aligned}
$$

One should not forget at this point that all the above relations were derived under the restriction that $|\kappa| \gg \frac{\partial \log \mathcal{G}_{0}\left(\mathbf{r}_{\alpha}, \mathbf{r}^{\prime} ; s\right)}{\partial \mathbf{n}_{\alpha}}$. This restriction is not particularly stringent if one does not approach the region $\kappa \cong 0$.

\section{Results and Discussion}

Taking now equations Eqs.20 and 27 and assuming that the surface density $\frac{M}{S_{\omega}}$ and the volume density $\frac{N}{V}$ are finite, we end up with the following form of the free energy in the limit of $\kappa>0$

$$
\begin{aligned}
\beta \mathcal{F} & \cong \beta \mathcal{F}_{0}-(N-M) \ln V-M \ln S_{\omega}+ \\
& +\left(\frac{N}{V}\right)\left[\frac{\sqrt{\mathcal{N}}}{\sqrt{\pi}} \int d S_{\omega}-\mathcal{N} \int d S_{\omega} \frac{1}{R_{\omega}}+\frac{2 \sqrt{\mathcal{N}}^{3}}{3 \sqrt{\pi}} \int d S_{\omega}\left(\frac{1}{R_{\omega}^{2}}+\frac{1}{R_{\omega 1} R_{\omega 2}}\right)\right]+ \\
& +\left(\frac{M}{S_{\omega}}\right)\left[\frac{4 \sqrt{\mathcal{N}}}{\sqrt{\pi}} \int d S_{\omega} \frac{1}{R_{\omega}}-\frac{\sqrt{\mathcal{N}}}{\kappa \sqrt{\pi}} \int d S_{\omega}\left(\frac{1}{R_{\omega}^{2}}-\frac{1}{R_{\omega 1} R_{\omega 2}}\right)-\mathcal{N} \int d S_{\omega} \frac{1}{R_{\omega 1} R_{\omega 2}}\right]- \\
& -\frac{\kappa}{2}\left(\frac{M}{S_{\omega}}\right)^{2}\left(\frac{N}{V}\right)^{-1}\left[\int d S_{\omega}+\frac{4 \sqrt{\mathcal{N}}}{\sqrt{\pi}} \int d S_{\omega} \frac{1}{R_{\omega}}-\mathcal{N} \int d S_{\omega} \frac{1}{R_{\omega 1} R_{\omega 2}}\right]+\ldots
\end{aligned}
$$

$\beta \mathcal{F}_{0}$ contains irrelevant constants and combinatorial terms, while the second and the third terms describe the removal of $M$ out of total of $N$ volume translational degrees of freedom and creation of $M$ surface translational degrees of freedom due to the existence of mobile surface sliplinks. The nature of the other terms in the free energy is also straightforwardly discernible (see below).

In the opposite limit of $\kappa<0$, assuming now that the two surface densities $\frac{M}{S_{\omega}}$ and $\frac{N}{S_{\omega}}$ are finite, we derive the following form of the free energy

$$
\begin{aligned}
\beta \mathcal{F} & \cong \beta \mathcal{F}_{0}-N \ln S_{\omega}+ \\
& +\left(\frac{N}{S_{\omega}}\right)\left[-\kappa^{2} \mathcal{N} \int d S_{\omega}+\frac{2}{\kappa} \int d S_{\omega} \frac{1}{R_{\omega}}-\mathcal{N} \int d S_{\omega}\left(\frac{1}{R_{\omega}^{2}}-\frac{1}{R_{\omega_{1}} R_{\omega_{2}}}\right)\right]- \\
& -\left(\frac{M}{S_{\omega}}\right) \frac{1}{\kappa^{2}} \int d S_{\omega} \frac{1}{R_{\omega}^{2}}-\frac{1}{2}\left(\frac{M}{S_{\omega}}\right)^{2}\left(\frac{N}{S_{\omega}}\right)^{-1} \frac{1}{\kappa^{2}} \int d S_{\omega} \frac{1}{R_{\omega}^{2}}+\ldots
\end{aligned}
$$


where the second term now simply signifies that in this limit the chains are mostly adsorbed to the surface as we assumed that there are no volume interactions between them.

The above two results should be compared with the canonical form of the membrane elastic energy [1]

$$
\mathcal{F}=\frac{1}{2} K_{c} \int d S_{\omega}\left(\frac{1}{R_{\omega}}-\frac{1}{R_{0}}\right)^{2}+K_{G} \int d S_{\omega} \frac{1}{R_{\omega 1} R_{\omega 2}}
$$

wherefrom one obtains the contribution of the polymer - surface interactions to the curvature modulus $\left(K_{c}\right)$, spontaneous curvature radius $\left(R_{0}\right)$, as well as to the modulus of Gaussian curvature $\left(K_{G}\right)$.

On comparison with results of Ref. [8], it is clear that the fourth term in the free energy Eq.29 corresponds to the statistically averaged contact excluded volume interaction of the polymer solution with the deformed boundary, as the limit $|\kappa| \gg 0$ assumed in deriving this result essentially reduces to the Dirichlet boundary condition. We shall referr to this term in the free energy as the contact term.

The term linear in $\frac{M}{S_{\omega}}$ is the only one that vanishes completely for a flat surface and is formally analogous to an effective polymer adsorption term [8]. As it is non - zero only for a rough surface it stemms from the "in plane bridging" (see Ref. [8]) provided by the segments of the polymers between different sliplinks. Obviously it tends to curve the surface towards the polymer rich side and thus acts in opposition to the contact term.

The last term in Eq.29, being of the second order in $\frac{M}{S_{\omega}}$, corresponds to the free energy of chain segments caught by the sliplinks and thus brought in the immediate vicinity of the repulsive surface. Its zero and first order curvature terms are both negative, reflecting the predominant influence of the deminished entropy over the interaction energy $(2 \kappa$ per link) of the chains meeting at the sliplink confined to the supporting surface. This term acts to expose a larger area to the polymer rich side, thus tending to curve the supporting surface away from it in concert with the contact term. It is also the only term in the energy expansion that does not effect the curvature modulus at all.

The final rescaling of the curvature modulus and spontaneous curvature in this limit then assume the form

$$
K_{c} \longrightarrow K_{c}+\frac{2}{3} k T \phi \frac{\mathcal{R}_{G}^{3}}{\sqrt{\pi}}-k T\left(\frac{M}{S_{\omega}}\right) \frac{\mathcal{R}_{G}}{\kappa \sqrt{\pi}}+\ldots
$$


and

$$
\frac{K_{c}}{R_{0}} \longrightarrow \frac{K_{c}}{R_{0}}+k T \phi \mathcal{R}_{G}^{2}\left(1+2 \kappa\left(\frac{M}{S_{\omega}}\right)^{2} \frac{\phi^{-2}}{\mathcal{R}_{G}}\right)-k T\left(\frac{M}{S_{\omega}}\right) \frac{4}{\sqrt{\pi}} \mathcal{R}_{G}+\ldots
$$

where we introduced the radius of gyration, which in appropriate units assumes the form $\mathcal{R}_{G}=\sqrt{\mathcal{N}}$ and the volume density of polymers $\phi=\frac{N}{V}$.

The free energy of the system in the limit of $\kappa \gg 0$ thus emerges as being composed of three decoupled terms: the surface energy of the bulk polymer solution due to the repulsive interaction of the polymers with the surface, the energy of those polymer chains that happen to join different sliplinks and lie in the plane of the unperturbed surface, and the surface energy of sliplinks themselves, since at the points of crosslinking different segments of the chains make contact with the (repulsive) surface. After relaxing the limit $\frac{M}{N} \ll 1$ we can presume, that only the last two mechanisms will remain in making a substantial contribution to the total free energy. Unfortunately the relaxing of this limit makes the whole calculation much more demanding, probably due to the fact that the system goes through a surface gelation transition.

In the case of attractive interactions between the chains and the surface the third term corresponds to the free energy of a polymer bound to a deformed surface with energy $\kappa$ per surface area. The first term in the parenthesis is simply the adsorption energy [12]. The term linear in curvature preferrs bending towards the polymer rich side and thus acts exactly in the opposite direction as the analogous term in the contact term Eq.29. This is also the only term in the whole free energy expression that is linear in curvature.

The rescaling of the curvature modulus obviously stemms in this case from three different mechanisms which interestingly enough all act in the direction of destabilizing the curvature modulus. The first contribution, being linear in $\frac{N}{S_{\omega}}$ comes from the adsorption energy and is of the same form as in the case when no pinning to sliplinks is present [8]. The second and third contributions, being linear and quadratic in $\frac{M}{S_{\omega}}$ respectively, are obviously due to the presence of surface - bound sliplinks. The linear term is due to the energy difference in the pinning at the sliplink as compared to the 'soft' adsorption while the third term is due to the energy difference of an adsorbed portion of the chain when it is pinned by two sliplinks, at the beginning and at the end, and when it is 'softly' adsorbed.

The final rescaling of the elastic properties of the "dressed" mem- 
brane can be in this limit cast into the form

$$
K_{c} \longrightarrow K_{c}-2\left(\frac{N}{S_{\omega}}\right) \mathcal{R}_{G}^{2}-2\left(\frac{M}{S_{\omega}}\right) \kappa^{-2}\left(1+\frac{1}{2}\left(\frac{M}{S_{\omega}}\right)^{2}\left(\frac{N}{S_{\omega}}\right)^{-2}\right)+\ldots
$$

and

$$
\frac{K_{c}}{R_{0}} \longrightarrow \frac{K_{c}}{R_{0}}+\left(\frac{N}{S_{\omega}}\right) \frac{2}{\kappa}+\ldots
$$

The modifications in the Gaussian modulus are important because they extend only over the area occupied by polymers and are thus position dependent (otherwise they would make no contribution to the equations determining the shape of the "dressed" membrane). In the case of repulsive polymer - membrane interactions, i.e. $\kappa>0$ we obtain the following form for the change in the Gaussian curvature modulus

$$
K_{G} \longrightarrow K_{G}+\phi \frac{2 \mathcal{R}_{G}^{3}}{3 \sqrt{\pi}}-\left(\frac{M}{S_{\omega}}\right)\left[\mathcal{R}_{G}^{2}-\frac{\mathcal{R}_{G}}{\kappa \sqrt{\pi}}\right]+\frac{\kappa}{2}\left(\frac{M}{S_{\omega}}\right)^{2} \phi^{-1} \mathcal{R}_{G}^{2}+\ldots
$$

Since the signs of different contributions in this case are not uniform there is no general statement that one could make regarding the proliferation of handles.

In the opposite case of attractive polymer - membrane interactions, $\kappa<0$, the Gaussian curvature modulus has a very simple dependence on the polymer parameters

$$
K_{G} \longrightarrow K_{G}+\left(\frac{N}{S_{\omega}}\right) \mathcal{R}_{G}^{2}+\ldots
$$

that in conjunction with Eq.34 favours the proliferation of handles. The last result is interesting also from the point of view of recent analysis of the modifications in the Gaussian curvature modulus of partially polymerized surfactant membranes [5]. Clearly for large negative values of $\kappa$ our problem is isomorphous to the problem of polymers embedded in the membrane. In case there are no interactions between embedded polymer chains the result derived by Kozlov and Helfrich [5] for this particular model system reduces exactly to Eq.37. 


\section{Figure Captions}

Fig.1 Schematic representation of the model system. Polymers are pinned to the supporting surface $\mathbf{r}_{\alpha}$ with sliplinks of functionality four that are mobile along the surface. The interaction of the chains between the sliplinks with the surface is short ranged and described with the phenomenological parameter $\kappa$. 


\section{References}

[1] E.A. Evans and R.Skalak, Mechanics and Thermodynamics of Biomembranes, (CRC Press, Boca Raton) (1980).

[2] B. T. Stokke, A. Mikkelsen, A. Elgsaeter, Euro. Biophys. J. 13 (1986) 203, 219.

B. T. Stokke, A. Mikkelsen, A. Elgsaeter, Biophys. J. 49 (1986) 319.

[3] D.H. Boal, Biophys.J. 67 (1994) 521.

D.H. Boal, U. Seifert and J.C. Shillcock, Phys.Rev.E 48 (1993) 4274.

D.H. Boal, U. Seifert and A. Zilker, Phys.Rev.Lett. 69 (1992) 3405.

[4] R. Everaers, I.S. Graham, E. Sackmann and M.J. Zuckermann, preprint (1994).

[5] M.M. Kozlov and W. Helfrich, Langmuir 9 (1993) 2761.

[6] P.-G. de Gennes, J.Phys.Chem. 94 (1990) 8407.

[7] D. Hone, H. Ji, P.A. Pincus, Macromol. 20 (1987) 2543.

H. Ji, D. Hone, Macromol. 21 (1988) 2600.

J.T. Brooks, C.M. Marques, M.E. Cates, J. Phys. II. 1 (1991) 673.

[8] R. Podgornik, Europhys. Lett. 21(1993) 245.

[9] K.F. Freed, Adv.Chem.Phys. 22 (1980) 1.

S. F. Edwards, K. F. Freed, J. Phys. C 3 (1970) 739,750,760.

[10] R. Balian, C. Bloch, Ann.Phys. 60 (1970) 401, 84 (1974) 559.

B. Duplantier, Physica A 168 (1990) 179.

[11] K.F. Freed, Renormalization Group Theory of Macromolecules (Wiley \& Sons, New York) (1987).

[12] E. Eisenriegler, Polymers near Surfaces (World Scientific, Singapore) (1993). 


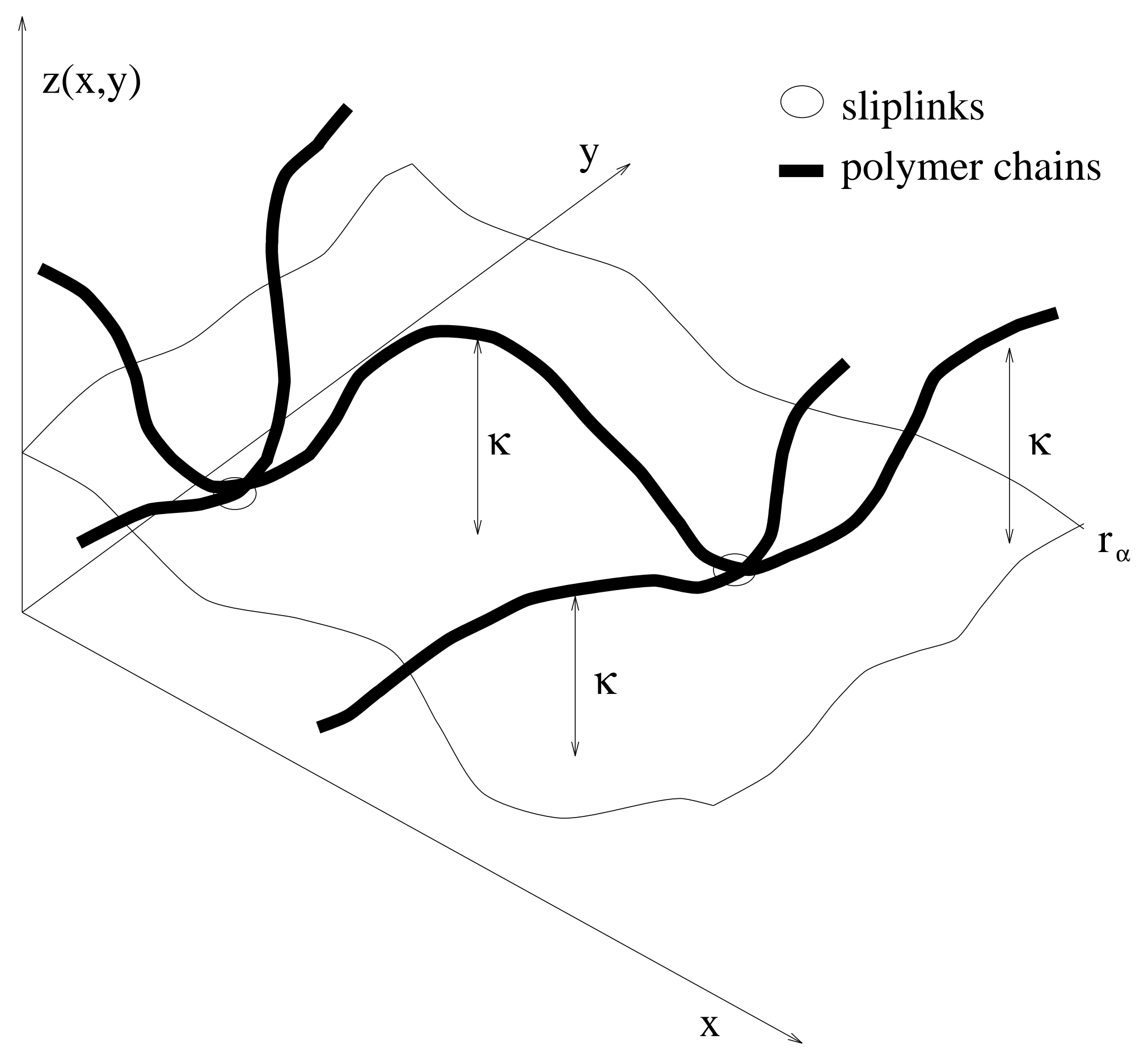

